\title{
Proposta de metodologia de dimensionamento para biodigestores tubulares
}

\author{
Design methodology proposal for tubular biodigesters \\ Propuesta de metodología de dimensionamiento para biodigestores tubulares
}

Recebido: 28/09/2021 | Revisado: 09/10/2021 | Aceito: 15/10/2021 | Publicado: 17/10/2021

Anelisiane Maria Alves
ORCID: https://orcid.org/0000-0003-1709-5256
Universidade Estadual do Oeste do Paraná, Brasil
E-mail: eng.anelisiane@ @mail.com
Jair Antonio Cruz Siqueira
ORCID: https://orcid.org/0000-0002-8140-444X
Eniversidade Estadual do Oeste do Paraná, Brasil
E-mail: jair.siqueira@ unioeste.br
Carlos Eduardo Camargo Nogueira
ORCID: https://orcid.org/0000-0003-3886-9118
Universidade Estadual do Oeste do Paraná, Brasil
E-mail: cecn1 @ yahoo.com.br
Eduardo Argou Aires Cunha
ORCID: https://orcid.org/0000-0001-6700-7037
Universidade Estadual do Oeste do Paraná, Brasil
E-mail: ed.argou@ @mail.com
Francisco Schmith Alves
ORCID: https://orcid.org/0000-0003-1366-8790
Universidade Estadual do Oeste do Paraná, Brasil
E-mail: franciscoschmith@ hotmail.com
Armin Feiden
ORCID: https://orcid.org/0000-0001-8068-5422
Universidade Estadual do Oeste do Paraná, Brasil
E-mail: armin.feiden@ @mail.com
Luciene Kazue Tokura

\section{Resumo}

Este trabalho compila as produções científicas presentes na literatura, que fundamenta a proposta de uma metodologia para dimensionamento técnico de biodigestores tubular. A metodologia proposta apresenta uma rotina de cálculo simplificada e organizada que permite ao usuário, com base em duas informações básicas (quantidade e classe que pertence o animal), obter como resultados os parâmetros geométricos da fossa do biodigestor e das caixas auxiliares. Em geral, o desenho do biodigestor é baseado em parâmetros geométricos definidos que levam em consideração o volume de resíduos gerados diariamente e o tempo que esses resíduos devem ser retidos para que ocorra a biodigestão. Esse volume é estimado com base nas necessidades energéticas de cada propriedade (produção de biogás), pelo número de animais presentes e na área disponível para aplicação do biofertilizante. As dimensões da fossa estimadas na metodologia foram o volume (metros cúbicos), a largura (metros), o comprimento (metros) e a altura útil (metros). A metodologia de dimensionamento foi apresentada sintetizada em um fluxograma. Por ter como dados primários o tipo e número de animais, o procedimento para o cálculo demanda uma adaptação regional de cada produtor, considerando a literatura disponível, com dados do tempo de retenção hidráulica e quantidade estimada de dejetos por dia.

Palavras-chave: Biodigestor tubular; Biodigestor de dimensionamento; Biogás.

\begin{abstract}
This work compiles the scientific productions present in the literature, which bases the proposal of a methodology for technical dimensioning of tubular biodigesters. The proposed methodology presents a simplified and organized calculation routine that allows the user, based on two basic information (quantity and class that the animal belongs to), to obtain as results the geometric parameters of the biodigester pit and auxiliary boxes. In general, the design of the biodigester is based on defined geometric parameters that consider the volume of residues generated daily and the
\end{abstract}


time that these residues must be retained for biodigestion to occur. This volume is estimated based on the energy needs of each property (biogas production), the number of animals present and the area available for application of the biofertilizer. The dimensions of the fossa estimated in the methodology were the volume (cubic meters), the width (meters), the length (meters) and the useful height (meters). The design methodology was presented synthesized in a flowchart. Having as primary data the type and number of animals, the procedure for the calculation requires a regional adaptation of each producer, considering the available literature, with data on hydraulic retention time and estimated amount of waste per day.

Keywords: Tubular biodigester; Biodigester dimensioning; Biogas.

\section{Resumen}

Este trabajo recopila las producciones científicas presentes en la literatura, que fundamenta la propuesta de una metodología para dimensionamiento técnico de biodigestores tubular. La metodología propuesta presenta una rutina de cálculo simplificada y organizada que permite al usuario, con base en dos informaciones básicas (cantidad y classe a la que pertenece el animal), obtener como resultados los parámetros geométricos de la fosa del biodigestor y de las cajas auxiliares. En general, el diseño del biodigestor se basa en parámetros geométricos definidos que toman en cuenta el volumen de residuos generados diariamente y el tiempo que estos residuos deben retenerse para que se produzca la biodigestión. Este volumen se estima en base a las necesidades energéticas de cada propiedad (producción de biogás), el número de animales presentes y el área disponible para la aplicación del biofertilizante. Las dimensiones de la fossa estimadas en la metodología fueron el volumen (metros cúbicos), la anchura (metros), la longitud (metros) y la altura útil (metros). La metodología de dimensionamiento fue presentada sintetizada en un diagrama de flujo. Por tener como datos primarios el tipo y número de animales, el procedimento para el cálculo requiere una adaptación regional de cada productor, considerando la literatura disponible, com datos sobre tiempo de retención hidráulica y cantidad estimada de desechos por día.

Palabras clave: Biodigestor tubular; Biodigestor de dimensionamiento; Biogás.

\section{Introdução}

Questões energéticas afetam diretamente o desenvolvimento humano, a economia global e o meio ambiente, tendo como discussão a substituição de fontes poluidoras por fontes renováveis (Vich \& Mansor, 2009; Costa Prates, 2005). No cenário brasileiro, a principal fonte de geração de energia elétrica é a hidráulica, seguida de gás natural, biomassa, derivados do petróleo, carvão mineral e derivados, nuclear e eólica. Já o consumo dessa oferta de energia elétrica é representado na ordem, pelo setor Industrial, Residencial, Perdas, comercial, público, energético, agropecuário e transportes (BEN, 2014).

Tais números devem ser encarados e analisados em sua especificidade, para um entendimento completo da realidade energética em questão. O setor agropecuário, por exemplo, apesar de representar apenas 4,3\% do consumo final de energia, apresentou uma crescente modernização, fato que provocou um aumento significativo na demanda energética e na quantidade de resíduos orgânicos (Portes, 2005).

Esse fenômeno pode ser verificado na própria Estrutura do Consumo no Setor Agropecuário entre 1975 e 2014 (BEN, 2014), onde a demanda de eletricidade e diesel teve um aumento substancial em concomitante com uma queda significativa no uso da lenha. Apesar de serem dados dos últimos 40 anos, esse balanço energético gradativo ocorre desde a década de 30 , quando o procedimento de modernização e implementação de inovações tecnológicas na agricultura brasileira teve início (Martine, 1991). Em concomitância com esse avanço tecnológico observou-se o aumento no consumo de energia, especialmente no início da década de noventa, indicando um maior emprego de máquinas e uma melhora nas condições da população rural, com o advento de aparelhos eletrodomésticos (Comitre, 1995).

Em decorrência a esses avanços, surgiram muitos problemas de ordem ambiental. Tanto com relação ao consumo de energia quanto à produção de resíduos com alto teor de substâncias patogênicas. Uma opção para resolver de forma eficiente estes problemas é conciliando a reciclagem controlada destes resíduos com a geração de energia. Essa reciclagem é feita por biodigestores que promovem uma digestão anaeróbica tendo como subprodutos um gás combustível com alto teor de $\mathrm{CH}_{4}$, denominado biogás e um lodo fertilizante que é o resíduo da matéria orgânica depois de fermentada, chamado biofertilizante (Portes, 2005). 
O sistema de biodigestão é uma estrutura projetada e construída de modo a produzir a situação mais favorável possível para que a degradação anaeróbia ocorra de forma em condições controladas de temperatura, umidade, homogeneidade e aeração. Sua estrutura é composta, de um modo geral, de duas partes, sendo uma a câmara de biodigestão, tanque fechado onde ocorre a fermentação anaeróbia da biomassa, e o corpo do biodigestor, juntamente com o gasômetro, onde o biogás é produzido e, fica retido para o seu devido aproveitamento ou conversão energética. Desde a década de 70, já existem diversos modelos propostos de biodigestor (Gaspar, 2003; Nascimento \& Lora, 2004; Linhares, 2008; Balmant, 2009; Cruz et al., 2019; Santos et al., 2020).

Conceitualmente, biomassa é o nome dado a qualquer matéria orgânica derivada de vegetais, como resultado do processo de conversão fotossintética. Tal derivação ocorre em função de uma reação entre gás carbônico $\left(\mathrm{CO}_{2}\right)$, água $\left(\mathrm{H}_{2} \mathrm{O}\right) \mathrm{e}$ luz solar, através do processo de fotossíntese que armazena uma fração de energia solar inicial nas ligações químicas de seus componentes finais (Souza et al., 2002). Já em âmbito energético, a chamada energia da biomassa é derivada de material vegetal e animal, resíduos de processos agrícolas, florestais e industriais e resíduos humanos ou animais (Bassam, 2010).

O processamento dessa biomassa para fins energéticos pode ocorrer de diversas formas, que são chamadas de rotas tecnológicas (Oliveira, 2005). A rota tecnológica cujo agente transformador é identificado como o biodigestor, pode apresentar como matéria-prima os resíduos orgânicos de origem agropecuária e como produto, o biogás (Torres \& Tarifa, 2012; Anjum, 2012; Coldebella, 2006).

Tais resíduos agropecuários são considerados um problema ambiental, uma vez que o Brasil possui uma representativa produção de resíduos gerados, sendo estimada, dentre outros, em cerca 105 milhões de m3 de dejetos líquidos de suínos e 7,8 milhões de toneladas de cama de aviário, que muitas vezes são despejados de forma incorreta, o que pode levar a contaminação de cursos d'água, situação que pode ser contornada quando a rota tecnológica de biodigestão é considerada. Entretanto, para essa alternativa, deverão ser considerados a criação confinada de animais, que proporciona a coleta adequada dos dejetos (Corrêa et al., 2011; EPE, 2015).

Já o biogás é composto, principalmente, por gás Carbônico $\left(\mathrm{CO}_{2}\right)$ e Metano $\left(\mathrm{CH}_{4}\right)$, com traços de Nitrogênio (N), Hidrogênio $(\mathrm{H})$ e gás Sulfídrico $\left(\mathrm{H}_{2} \mathrm{~S}\right)$ (Gaspar, 2003). Esse produto energético é resultante da ação digestiva de bactérias metanogênicas que agem por meio do agente de conversão que promove a biodigestão em condições anaeróbicas do material orgânico (Abreu Neto, 2007; Kunz et al., 2005).

Ao final do processo de conversão energética, a biomassa fermentada se encontra em forma líquida e rica em material orgânico, com grande poder de fertilização (Gaspar, 2003). O material digerido no biodigestor possui inúmeras aplicações como biofertilizante devido aos seus benefícios que proporcionam, entre outras coisas, uma melhor estrutura e atividade microbiológica, maior retenção de umidade, fornecimento de nutrientes minerais que melhoram a fertilidade do solo (Sganzerla, 1983; Machado, 2013).

\section{Metodologia}

A metodologia de estudo para o dimensionamento do biodigestor foi baseada nos parâmetros geométricos, levando em consideração o volume de dejetos gerado diariamente e o tempo de retenção hidráulica para a produção de biogás e biofertilizante. Posteriormente seguiu as metodologias de Von Sperling (1996), Feiden et al. (2004), Oliveira and Higarashi (2006), Kuczman (2007), Oliver et al. (2008), Gerscovich (2009), Lima (2011) e Peruzzo (2013).

O modelo tubular (Figura 1) se caracteriza por sua geometria horizontal, com largura maior que a profundidade. Umas de suas características técnicas mais marcantes diz respeito à produção de biogás, pois seu formato promove uma grande área de exposição ao sol, favorecendo a ação das bactérias que compõem o processo de digestão no interior do equipamento. Esse modelo é constituído pelas duas principais partes, sendo uma cúpula de plástico maleável, tipo PVC, que infla conforme se 
produz o gás e uma caixa de carga feita em alvenaria, podendo ser enterrada ou não (Morais, 2012; Moreira et al., 2014).

Figura 1. Modelo tubular de biodigestor.

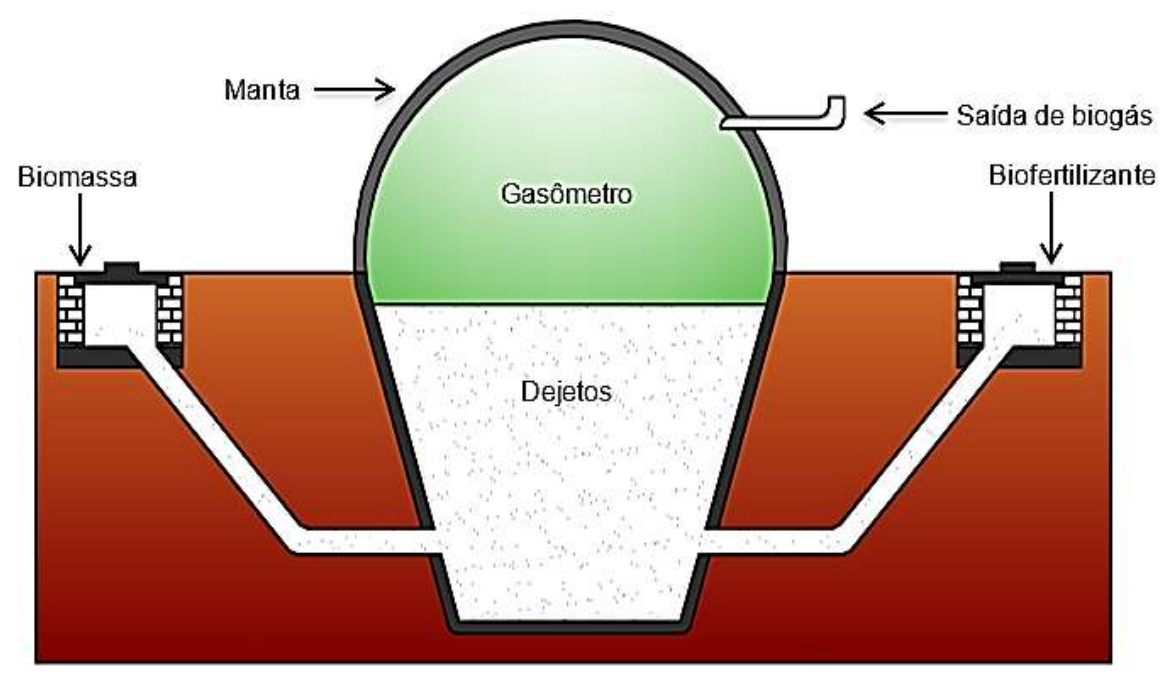

Fonte: Adaptada de Moreira et al. (2014).

Quanto ao seu funcionamento, o fluxo de substrato é contínuo, sendo introduzida em uma das extremidades do biodigestor (caixa de entrada), passando através do mesmo e sendo posteriormente descarregada na outra extremidade (caixa de saída). Esse processo ocorre pelo princípio físico de um embolo, sem misturas longitudinais, ou seja, as partículas mantêm sua identidade e permanecem no tanque por um período igual ao tempo de retenção hidráulica. Para que essa característica técnica seja garantida, umas das condições construtivas desse modelo de biodigestor é que sua estrutura apresente um valor alto na relação comprimento/largura (Von Sperling, 2016).

Dessa forma, o dimensionamento do biodigestor, é baseado em parâmetros geométricos definidos em função do volume de dejetos gerado diariamente e do tempo que esse dejeto deverá ficar retido no biodigestor para que a biodigestão aconteça. $\mathrm{O}$ volume do biodigestor deve estar de acordo com as necessidades energéticas da propriedade, com a capacidade de consumo do biogás produzido, com o número de animais existentes e com a área disponível para aplicação do biofertilizante. Posteriormente, deve-se seguir os procedimentos determinados por Oliver et al. (2008):

Deve-se calcular o volume diário de dejetos através da equação 1 , onde, $\mathrm{V}_{\mathrm{D}}=$ Volume de Dejetos; $\mathrm{N}=$ Número de animais e $\mathrm{D}_{\mathrm{A}}=$ Quantidade de dejetos por animal (L/dia);

$$
V_{D}=D_{A} \times N
$$

Com o Volume diário de dejetos, calculou-se a proporção e água necessária para a mistura (equação 2), onde VA = Volume de água, $\mathrm{V}_{\mathrm{D}}=$ Volume de Dejetos diário e $\mathrm{R}_{\mathrm{A}}=$ Fator de relação dejeto e água;

$$
V_{A}=V_{D} \times R_{A}
$$

Calculou-se posteriormente, o volume da carga diária produzida (equação 3), onde $\mathrm{V}_{\mathrm{C}}=$ Volume da carga diária $\left(\mathrm{m}^{3}\right)$; $\mathrm{V}_{\mathrm{A}}=$ Volume de água e $\mathrm{V}_{\mathrm{D}}=$ Volume de Dejetos diário; 


$$
V_{C}=\left(V_{A}+V_{D}\right)
$$

O cálculo do Volume total da fossa do biodigestor é realizado pela equação 4 , onde VFB = Volume da fossa do biodigestor $\left(\mathrm{m}^{3}\right) ; \mathrm{V}_{\mathrm{C}}=$ Volume da Carga diária (m³/dia) e TRH = Tempo de retenção hidráulica (dias):

$$
V_{F B}=V_{C} \times T R H
$$

O TRH, representa o tempo que o efluente deve permanecer na câmara para atingir a degradação esperada e é um parâmetro que depende da capacidade das bactérias em degradar a matéria orgânica, ou seja, vai depende do tipo de dejeto inserido no biodigestor (Oliveira \& Higarashi, 2006).

$\mathrm{O}$ VFB encontrado em função do $\mathrm{V}_{\mathrm{C}}$ e TRH deve ser equivalente ao volume geométrico $\left(\mathrm{V}_{\mathrm{G}}\right)$ da fossa de forma trapezoidal. Dessa forma, considera-se que $\mathrm{V}_{\mathrm{G}}$ é calculado através da equação 5, onde, a e b são as medias da base superior e inferior, respectivamente, do trapézio que forma a seção transversal da fossa, $\mathrm{h}=$ altura útil da fossa e $\mathrm{L}=$ comprimento longitudinal da fossa (Figura 2).

$$
V_{F B}=\frac{(a+b)}{2} \times h \times L
$$

Figura 2. Modelo tubular.

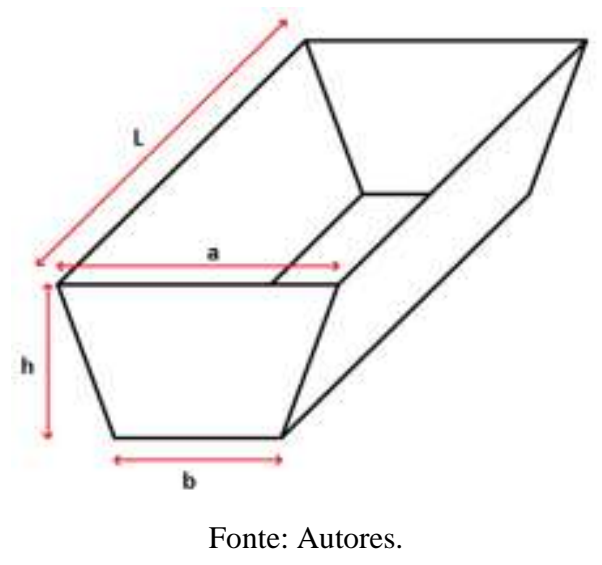

Conforme apresentado, o volume geométrico da fossa, obtido em função do volume de carga diária e do tempo de retenção hidráulica, quando aplicado na equação 5, obtém-se três incógnitas, que representam as medidas gerais da fossa do biodigestor. Para determinar os valores referentes a tais medidas, deve-se levar em conta, condições de funcionamento do sistema de biodigestão, como a inclinação correta do talude, a proporção entre largura e comprimento da superfície da fossa e a altura útil (Gerscovich, 2009; Von Sperling, 1996; Feiden et al., 2004). Para o desenvolvimento dos cálculos, optou-se por considerar o ângulo de inclinação do talude equivalente a $110^{\circ}$ como referência média para taludes. A partir dessa definição, é possível montar relações entre as medidas da seção do biodigestor.

A seção da fossa do biodigestor (Figura 3), é representada por um trapézio cujas medidas são denominadas, como sendo as variáveis a (largura da base superior), b (largura da base inferior) e h (altura). Nesse trapézio, ao se isolar um dos cantos inclinados, forma-se um triângulo retângulo cujo ângulo adjacente à altura (h) foi denominado $\theta$, e equivale a subtração do ângulo reto da inclinação do talude. 
Figura 3. Seção da fossa.

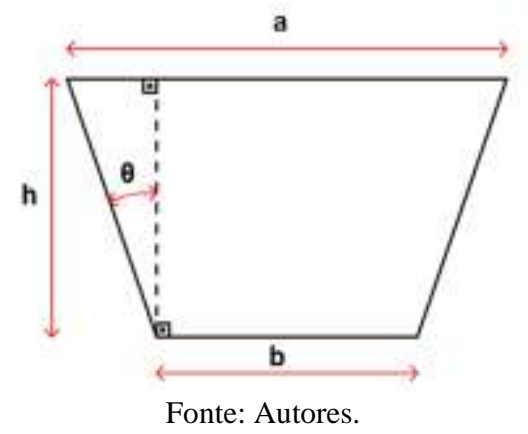

Isolando o triângulo retângulo na seção trapezoidal da fossa (Figura 4), são obtidas novas relações entre as medidas a (largura da base superior), b (largura da base inferior) e h (altura).

Figura 4. Inclinação do talude.

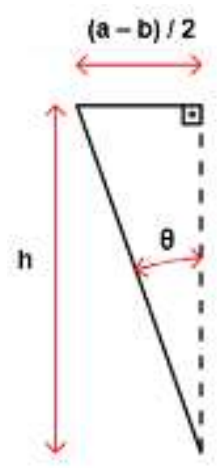

Fonte: Autores.

Considerando a inclinação inicial do talude igual a $110^{\circ}$ (Gerscovich, 2009; Von Sperling, 1996; Feiden et al., 2004), obtém-se que o valor de $\theta$ (equação 6) é equivalente a $20^{\circ}$ (equação 7). Baseando-se nesses parâmetros, pode ser obtida a equação (8), pela função tangente, cuja igualdade é representada pela divisão do cateto oposto pelo cateto adjacente:

$$
\begin{aligned}
& \tan \theta=\frac{\frac{(a-b)}{2}}{h} \\
& \tan 20=\frac{(a-b) \times h}{2} \\
& a=0,7279 h+b
\end{aligned}
$$

A próxima relação a ser determinada, são as dimensões de largura (a) e comprimento (L) da superfície superior da fossa (Figura 3). Para os biodigestores tubulares, deve ser respeitada a relação largura/comprimento usual que pode variar de 1:3 (Lima, 2011) a 1:5 (Feiden et al., 2004), já que a proporção diz respeito a manutenção das características do fluxo hidráulico (tipo pistão) nesse modelo de biodigestor (Kuczman, 2007).

A escolha da proporção foi de 1:5 para representar a relação entre a largura (a) e comprimento (L) da superfície superior da fossa, conforme equação 9 . 


$$
L=5 a
$$

Aplicando a equação 8 na equação 9, obtém a equação 10:

$$
L=5 \times(0,7279 h+b) \rightarrow L=3,6395 h+5 b
$$

Prossegue-se então, com a substituição de duas Incógnitas na equação 5, onde os termos 'a' (largura superior da fossa) e 'L' (comprimento da fossa), são substituídos pela equivalência das equações 8 e 10, respectivamente, obtendo-se assim a equação 11:

$$
V_{P B}=\frac{(0,7279 h+b+b)}{2} \times h \times(3,6395 h+5 b) \quad \rightarrow \quad 1,3245 h^{3}+5,459 b h^{2}+5 h b^{2}-V_{P B}=0
$$

No item anterior, ao prosseguir a substituição, operações e isolar os termos semelhantes, obteve-se uma igualdade envolvendo a altura da fossa (h), a largura da superfície inferior da fossa (b) e o Volume da fossa do Biodigestor ( $\mathrm{V}_{\mathrm{FB}}$ ).

Ao observar a equação 11, nota-se que estando determinado os valores da altura e do volume da fossa do biodigestor, a única incógnita restante é a largura da superfície inferior da fossa, representando uma equação de $2^{\circ}$ grau (Equação 12).

$$
5 h b^{2}+5,459 h^{2} b+1,3245 h^{3}-V_{F B}=0
$$

Onde: $\mathrm{b} \rightarrow$ incógnita da equação; $5 \mathrm{~h} \rightarrow$ primeiro termo numérico da equação; $5,459 \mathrm{~h}^{2} \rightarrow$ segundo termo numérico da equação; $1,3245 \mathrm{~h}^{3}-\mathrm{V}_{\mathrm{FB}} \rightarrow$ terceiro termo da equação.

A equação de $2^{\circ}$ grau resultante da relação entre altura, largura da superfície inferior e o volume da fossa do biodigestor pode ser resolvida através do método de Bhaskara (Peruzzo, 2013) representado pela equação 13.

$$
b=\frac{-y \pm \sqrt{y^{2}-4 x z}}{2 x}
$$

A resolução da equação de $2^{\circ}$ grau (equação 12), permite encontrar a um par de possíveis soluções para a incógnita, nesse caso, a largura da superfície inferior da fossa (b), sendo um valor negativo e um positivo. Como nessa situação, a incógnita trata-se de uma medida real, descarta-se de antemão a possível solução com valor negativo:

Em seguida, aplica-se os termos numéricos da equação 12 na equação 13, obtendo-se a equação 14:

$$
b=\frac{-5,459 h^{2}+\sqrt{\left(5,459 h^{2}\right)^{2}-4\left(5 h \times\left(1,3245 h^{3}-V_{F B}\right)\right)}}{2 \times(5 h)} \quad \rightarrow \quad b=\frac{-5,459 h^{2}+\sqrt{\left.29,80 h^{2}-26,49 h^{3}+20 h V_{F B}\right)}}{10 h}
$$

Dessa forma, as equações 8, 10 e 14 permitem determinar, respectivamente, os valores da largura da superfície superior (a), o comprimento e a largura da superfície inferior (b) da fossa do biodigestor. As equações dependem de um valor pré-determinado para a altura $(\mathrm{h})$ e para o volume $\left(\mathrm{V}_{\mathrm{FB}}\right)$. $\mathrm{O} \mathrm{V}_{\mathrm{FB}}$ é determinado pela equação 5, entretanto, o valor da altura (h) deve ser definido arbitrariamente. 
Quanto à altura, os valores variam de 1,50 a 4,50 m para biodigestores tubulares e para o dimensionamento das lagoas anaeróbias, de 1,50 a 3,00 m, conforme dados disponíveis na literatura (Silva, s.d.). Para Volumes com até $100 \mathrm{~m}$, a definição para a altura ideal é de $1,5 \mathrm{~m}$; entre 100 e $500 \mathrm{~m}^{3}$, de 2,5 m; entre 500 e $2000 \mathrm{~m}^{3}$, de $3,5 \mathrm{~m}$ e acima de $2000 \mathrm{~m}^{3}$, de 4,5 m.

Os elementos complementares considerados nessa seção são as caixas de entrada e saída (Figura 5). A Caixa de entrada deve ser equivalente ao Volume da Carga diária (VC), acrescido de $20 \%$ e a Caixa de saída, equivalente a três vezes o volume da caixa de entrada $\left(\mathrm{em} \mathrm{m}^{3}\right)$. As caixas deverão ser de seção horizontal quadrada e com profundidade útil definida em $1 \mathrm{~m}$. Os cálculos podem ser obtidos pelas equações 15, 16, 17 e 18 .

Figura 5. Representação genérica para caixas de entrada e saída.

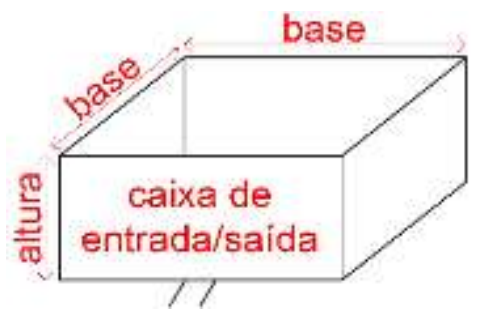

Fonte: Autores.

$$
\begin{aligned}
& \mathrm{V}_{\mathrm{CE}}=\mathrm{V}_{\mathrm{C}} \times 1,20=\mathrm{P}_{\mathrm{E}} \times \mathrm{L}_{C E^{2}} \\
& \mathrm{P}_{\mathrm{E}}=1,00 ; \quad \mathrm{L}_{\mathrm{CE}}=\sqrt{V_{C E}} \\
& \mathrm{~V}_{\mathrm{CS}}=\mathrm{V}_{\mathrm{CE}} \times 3 \\
& \mathrm{P}_{\mathrm{S}}=1,00 ; \quad \mathrm{L}_{\mathrm{CS}}=\sqrt{V_{C S}}
\end{aligned}
$$

Onde: $\mathrm{V}_{\mathrm{CE}}=$ Volume da caixa de entrada $; \mathrm{V}_{\mathrm{CS}}=$ Volume da caixa de saída; $\mathrm{P}_{\mathrm{E}}=$ Profundidade da caixa de entrada; $\mathrm{P}_{\mathrm{S}}=$ Profundidade da caixa de saída; $\mathrm{L}_{\mathrm{CE}}=$ Lateral da caixa de entrada; $\mathrm{L}_{\mathrm{CS}}=$ Lateral da caixa de saída.

\section{Resultados e Discussão}

As dimensões a serem estimadas foram o Volume da fossa $\left(\mathrm{V}_{\mathrm{F}}\right)$, largura superior da fossa $(\mathrm{a})$, largura inferior da fossa (b), comprimento da fossa (L) e altura útil da fossa (h) (Figura 2). A metodologia de dimensionamento é apresentada resumida no fluxograma abaixo (Figura 6). 
Figura 6. Fluxogramas de dimensionamento.

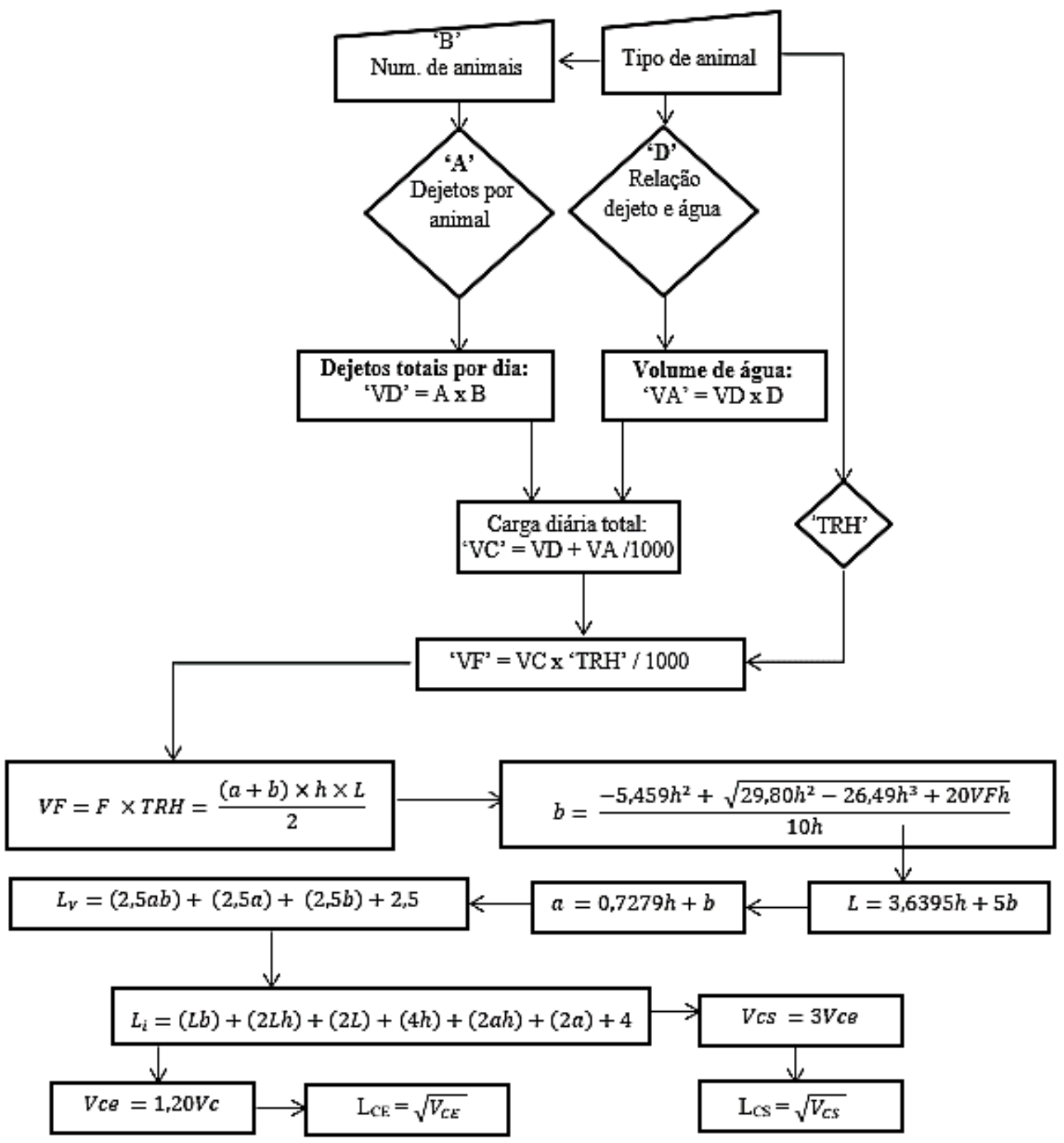

Fonte: Autores.

O fluxograma apresentado nos resultados apresenta um resumo dos procedimentos metodológicos de dimensionamento técnico do biodigestor, onde são necessárias as observações a seguir.

O Volume de dejetos por dia $\left(V_{D}\right)$ é equivalente a multiplicação da quantidade de animais pelo fator de $V_{D}(L /$ dia), obtido na Tabela 1. Sendo possível a soma de mais que um tipo de animal no cálculo. O Volume de água também é calculado em função da multiplicação do $V_{D}$ por um fator obtido na Tabela 1 de acordo com o tipo de animal (Macedo, 2013; Bond, 2015; Kohler, 2017). 
Tabela 1. Dados primários.

\begin{tabular}{lccc}
\hline \multicolumn{1}{c}{ Tipos de animais } & Fator relação dejeto/água & TRH (dias) & Dejetos (L/dia) \\
\hline Aves & $1: 3$ & 60 & 0,18 \\
Caprinos & $1: 5$ & 45 & 5 \\
Bovinos & $1: 1$ & 30 & 10 \\
Equinos & $1: 1$ & 45 & 10 \\
Suínos machos (cachaço) & $1: 2$ & 30 & 9 \\
Suínos fêmeas em lactação & $1: 2$ & 30 & 27 \\
Leitões na creche & $1: 2$ & 30 & 1,4 \\
Bovinos em lactação & $1: 1$ & 30 & 30 \\
Bezerros & $1: 1$ & 30 & 2 \\
Bois de corte & $1: 1$ & 30 & 15 \\
\hline
\end{tabular}

Fonte: Macedo (2013), Bond (2015) e Kohler (2017).

O VF é equivalente a multiplicação do VC pelo tempo que essa carga deverá ficar retida no biodigestor, ou seja, o tempo de retenção hidráulica (TRH). Também é aplicado um fator de conversão de unidade de medidas, visando transformar em metro cúbico $\left(\mathrm{m}^{3}\right)$ o volume que está em litros $(\mathrm{L})$. O TRH, também é obtido na Tabela 1 em função do tipo de animal (Macedo, 2013; Bond, 2015; Kohler, 2017).

De posse dos dados acima descritos, é possível dar início a rotina de cálculo desenvolvida nesse trabalho e obter como resultado os parâmetros geométricos do biodigestor.

\section{Conclusão}

Nesse trabalho, após uma revisão de literatura, foi proposta uma metodologia de dimensionamento técnico para biodigestores modelo tubular, evidenciando as inúmeras variáveis envolvidas no processo.

A metodologia proposta apresenta uma rotina de cálculos que foi simplificada e organizada com base em duas informações básicas (quantidade e tipo de animal), de forma que o usuário consegue obter como resultado parâmetros geométricos da fossa do biodigestor e das caixas de carga e descarga.

No entanto, deve-se observar que por ter como dados primários o tipo e a quantidade de animais, o processo de cálculo demanda uma adaptação regional de cada produtor, que deve levar em conta a literatura disponível abordando e indicando os valores de Tempo de Retenção Hidráulica e a produção estimada de dejetos por dia.

Sugere-se para trabalhos futuros elaborar um sistema computacional para dimensionamento do biodigestor tubular.

\section{Referências}

Abreu Neto, M. S. (2007). Tratamento de águas residuárias de suinocultura em reator anaeróbio compartimentado seguido de reator UASB. Dissertação de mestrado. Universidade Estadual Paulista, Faculdade de Ciências Agrárias e Veterinárias, Jaboticabal. 170p.

Anjum, A. (2012). Biomass: Energy and Environmental Concerns in Developing Country. I Research Journal of Environment Sciences, 1 (1), $54-57$.

Balmant, W. (2009). Concepção, construção e operação de um biodigestor e modelagem matemática da biodigestão anaeróbica. Dissertação de mestrado. Pós-Graduação em Engenharia e Ciência dos Materiais - PIPE. Setor de Tecnologia, Universidade Federal do Paraná, Curitiba. 59p.

Bassam, N. (2010). Handbook of Bioenergy Crops: A Complete Reference to Species, Development and Applications. Earthscan: The United Kingdom, 2010. $<$ https://nishat2013.files.wordpress.com/2013/11/handbook-of-bioenergy-crops.pdf >.

BEN. (2015). Balanço Energético Nacional 2015: Ano base 2014/Empresa de Pesquisa Energética. EPE, 2015. https://ben.epe.gov.br/downloads/Relatorio_Final_BEN_2015.pdf. 
Bond, W. E. (2015). Biodigestor. Apresentação BGS - Equipamentos para biodigestores. In: XIX Seminário nordestino de Agropecuária, 2015, Fortaleza.

Coldebella, A. (2006). Viabilidade do uso do biogás da bovinocultura e suinocultura param geração de energia elétrica e irrigação em propriedades rurais. Dissertação de mestrado. Engenharia Agrícola/Engenharia de Sistemas Agroindustriais, Universidade Estadual do Oeste do Paraná, Cascavel.

Comitre, V. (1995). A questão energética e o padrão tecnológico da agricultura brasileira. Informações Econômicas, 25 (12), $29-34$.

Corrêa, J. C., Benites, V. M., \& Rebellatto, A. (2011). O uso dos resíduos animais como fertilizantes. In: II Simpósio Internacional sobre Gerenciamento de Resíduos Agropecuários e Agroindustriais, Foz do Iguaçu, PR.

Costa, R. C., \& Prates, C. P. T. (2005). O papel das fontes renováveis de energia no desenvolvimento do setor energético e barreiras à sua penetração no mercado. BNDES Setorial, 21, 5-30, http://www.bndes.gov.br/SiteBNDES/export/sites/default/bndes_pt/Galerias/Arquivos/conhecimento/bnset/set2102.pdf.

Cruz, H. M., Barros, R. M., Santos, I. F. S., \& Tiago Filho, G. L. (2019). Estudo do potencial de geração de energia elétrica a partir do biogás de digestão anaeróbica de resíduos alimentares. Research, Society and Development, 8 (5), e3785811. 10.33448/rsd-v8i5.811.

EPE. (2015). Empresa de Pesquisa Energética. Inventário Energético de Resíduos Rurais. Ministério de Minas e Energia.

Feiden, A., Reichl, J., Schwab, J., \& Schwab, V. (2004). Avaliação da eficiência de um biodigestor tubular na produção de biogás a partir de águas residuárias de suinocultura. In: AGRENER GD 2004. $5^{\circ}$ Encontro de Energia no Meio Rural e geração Distribuída. 2004. Anais. Campinas: Núcleo Interdisciplinar de Planejamento Energético da UNICAMP/NIPE. 2004.

Gaspar, R. M. B. L. (2003). Utilização de biodigestores em pequenas e médias propriedades rurais com ênfase na agregação de valor: um estudo de caso na região de Toledo-PR. Dissertação de mestrado. Engenharia de Produção e Sistemas. Universidade Federal de Santa Catarina, Florianópolis.

Gerscovich, D. M. S. (2009). Apostila Estabilidade de Taludes. Faculdade de Engenharia/UERJ. Departamento de Estruturas e Fundações.

Kohler, P. (2017). Biodigestor. Apresentação Biokohler-Biodigestores. Show rural Coopavel. Cascavel.

Kuczman, O. (2007). Tratamento anaeróbio de efluente de fecularia em reator horizontal de uma fase. Dissertação de mestrado. Engenharia Agrícola, Universidade Estadual do Oeste do Paraná, Cascavel, PR.

Kunz, A., Higarashi, M. M., \& Oliveira, P. A. (2005). Tecnologias de manejo e tratamento de dejetos de suínos estudadas no Brasil. Cadernos de Ciência \& Tecnologia, 22 (3), 651-665.

Lima, H. Q. (2011). Avaliação dos modelos Hashimoto e AMS-III.D para produção de metano com dejetos de suínos. Dissertação de mestrado. Tecnologia de Alimentos, Universidade Federal do Paraná, Curitiba. 148p.

Linhares, A. C. S. (2008). Análise da presença de um enfoque ambientalista em uma escola /faculdade de tecnologia na cidade de Curitiba. Um estudo de caso baseado na ISO 14001. Dissertação de mestrado. Engenharia Química, Pontifícia Universidade Católica do Paraná, Curitiba.

Macedo, F. J. (2013). Dimensionamento de biodigestores para tratamento de dejetos da produção suína. Graduação em Engenharia Sanitária e Ambiental, Universidade Federal de Santa Catarina, Florianópolis-SC.

Machado, G. B. (2013). Fertilizantes. Portal resíduos sólidos. Offenbach, Alemanha. http://www.portalresiduossolidos.com.

Martine, G. (1991). A trajetória da modernização agrícola: A quem beneficia? In: Lua Nova, 23, 7-37. http://www.scielo.br/pdf/ln/n23/a03n23.pdf.

Morais, M. A. (2012). Estudo experimental e avaliação econômica da operação de biodigestores tubulares para a produção de biogás a partir de resíduos da suinocultura. Dissertação de mestrado. Engenharia de energia, Universidade Federal de Itajubá. 92p.

Moreira, T. S., Carvalho, R. F., Cassiano, E. C. O., Vazquez, D. C. Z., Nogueira, R. G. S., Paucar, L. C., Perna Junior, F., \& Rodrigues, P. H. M. (2014). Utilização de biodigestores como alternativa para o tratamento de dejetos oriundos da produção animal. In: Novos desafios da pesquisa em nutrição $e$ produção animal. Pirassununga-SP.

Nascimento, M. A. R., \& Lora, E. E. S. (2004). Geração termelétrica: planejamento, projeto e operação. Interciência. 631p.

Oliveira, L. R. P. (2005). Biodigestor. In: VII Simpósio Goiano de Avicultura e II Simpósio Goiano de Suinocultura, 2005, Goiânia: Avesui Centro-Oeste, p. 4-8.

Oliveira, P. A., \& Higarashi, M. (2006). Geração e utilização do biogás em unidades de produção de suínos. Concórdia: Embrapa Suínos e Aves. PNMA II Programa Nacional do Meio Ambiente. $41 \mathrm{p}$

Oliver, A. P. M., Souza Neto, A. A., Quadros, D. G., \& Valladares, R. R. (2008). Manual de treinamento em biodigestão. Agência dos Estados Unidos para o Desenvolvimento Internacional (USAID). IRES (Market Development for Biodigestion in Brazil). Instituto Winrock - Brasil, 2008. 23p.

Peruzzo, J. (2013). Evolução dos métodos de resolução de equações algébricas. Santa Catarina: Clube de Autores. 129p.

Portes, Z. A. (2005). Aplicativo computacional para projetos de biodigestores rurais. Dissertação de mestrado. Universidade Estadual Paulista Júlio de Mesquita Filho, Botucatu.

Santos, C. A. A. S., Cutrim Jr, C. F., Oliveira, L. L., Silva, P. H. F., Maia, I. C. C. D., Nascimento, B. L. M., Serra, M. A. A. O., \& Façanha Filho, P. F. (2020). Biodigestor anaeróbico para produção sustentável de biogás em propriedade rural da cidade de Açailândia, Maranhão, brasil. Research, Society and Development, 9 (7), e362974262. 10.33448/rsd-v9i7.4262

Sganzerla, E. (1983). Biodigestor: uma solução. Agropecuária. 86p. 
Research, Society and Development, v. 10, n. 13, e407101321172, 2021

(CC BY 4.0) | ISSN 2525-3409 | DOI: http://dx.doi.org/10.33448/rsd-v10i13.21172

Silva, C. E. (S. D.). Tratamento de Resíduos e Impactos Ambientais. Notas de Aula. Departamento de Hidráulica e Saneamento. Universidade Federal de Santa Maria. [S. D.]. http://jararaca.ufsm.br/

Souza, S. N. M., Sordi, A., \& Oliva, C. A. (2002). Potencial de energia primária de resíduos vegetais no Paraná. In: Encontro de Energia no Meio Rural, abr., 2002, Campinas. http://www.feagri.unicamp.br/energia/agre2002/pdf/0052.pdf.

Torres, G. A., \& Tarifa, L. R. M. (2012). Aproveitamento de Resíduos Agrícolas. In: Dossiê Técnico. Universidade de São Paulo,

Vichi, F. M., \& Mansor, M. T. C. (2009). Energia, meio ambiente e economia: o Brasil no contexto mundial. Química Nova, 32 (3), 757-767. 10.1590/S010040422009000300019 .

Von Sperling, M. (2016). Princípios básicos do tratamento de esgotos. Belo Horizonte: Departamento de Engenharia Sanitária e Ambiental - Universidade Federal de Minas Gerais. 211p. il. 